\title{
An Objectivist Argument for Thirdism
}

\author{
The OSCAR Seminar: Adam Arico, Nathan Ballantyne, Matt Bedke, \\ Jacob Caton, Ian Evans, Don Fallis, Brian Fiala, Martin Frické, \\ David Glick, Peter Gross, Terry Horgan, Jenann Ismael, John Pollock, \\ Daniel Sanderman, Paul Thorn, Orlin Vakarelov \\ Department of Philosophy \\ University of Arizona \\ Tucson, Arizona 85721
}

The literature on the Sleeping Beauty problem has been dominated by Bayesians. ${ }^{1}$ Even those authors who are not Bayesians ${ }^{2}$ have addressed the problem without using much of the rich machinery available to objective probability theorists. We show that the objective probability theorist has a very simple argument for thirdism.

\section{Objective Probability}

Bayesians take "definite" or "single-case" probabilities to be basic. Definite probabilities attach to closed formulas or propositions. We write them here using small caps: $\operatorname{PROB}(P)$ and $\operatorname{PROB}(P / Q)$. Most objective probability theories begin instead with "indefinite" or "general" probabilities (sometimes called "statistical probabilities"). Indefinite probabilities attach to open formulas or propositions. We write indefinite probabilities using lower case "prob" and free variables: $\operatorname{prob}(B x / A x)$. The indefinite probability of an $A$ being a $B$ is not about any particular $A$, but rather about the property of being an $A$. In this respect, its logical form is the same as that of relative frequencies. For instance, we might talk about the probability of a human baby being female. That probability is about human babies in general - not about individuals. If we examine a baby and determine conclusively that she is female, then the definite probability of her being female is 1 , but that does not alter the indefinite probability of human babies in general being female.

Most objective approaches to probability tie probabilities to relative frequencies in some way, and the resulting probabilities have the same logical form as the relative frequencies. That is, they are indefinite probabilities. The simplest theories identify indefinite probabilities with relative frequencies. ${ }^{3}$ It is often objected that such "finite frequency theories" are inadequate because our probability judgments often diverge from relative frequencies. For example, we can talk about a coin being fair (and so the indefinite probability of a flip landing heads is 0.5) even when it is flipped only once and then destroyed (in which case the relative frequency is either 1 or 0 ). For understanding such indefinite probabilities, it has been suggested that we need a notion of probability that talks about possible instances of properties as well as actual instances. Theories of this sort are sometimes called "hypothetical frequency theories". C. S. Peirce was perhaps the first to make a suggestion of this sort. Similarly, the statistician R. A. Fisher, regarded by many as "the father of modern statistics", identified probabilities with ratios in a "hypothetical infinite population, of which the actual data is regarded as constituting a random sample" (1922, p. 311). Karl Popper (1956, 1957, and 1959) endorsed a theory along these lines and called the resulting probabilities propensities. Henry Kyburg (1974a) was the first to construct a precise version of this theory (although he did not endorse the theory), and it is to him that we owe the name "hypothetical frequency theories". Kyburg (1974a) also insisted that von Mises should also be considered a hypothetical frequentist. More recent attempts to formulate precise versions of what might be regarded as hypothetical frequency theories are van Fraassen (1981), Bacchus (1990), Halpern (1990), Pollock (1990), Bacchus et al (1996).

It has always been acknowledged that for practical decision-making we need definite probabilities rather than indefinite probabilities. So theories that take indefinite probabilities as basic

\footnotetext{
1 Elga 2000, Lewis 2001, Dorr 2002, Arntzenius 2003, Bradley 2003, Hitchcock 2004, Monton 2002, Weintraub 2004, White 2006

2 For instance, Horgan 2004, 2007.

3 Examples are Russell 1948; Braithwaite 1953; Kyburg 1961, 1974; Sklar 1970, 1973. William Kneale (1949) traces the frequency theory to R. L. Ellis, writing in the 1840's, and John Venn (1888) and C. S. Peirce in the 1880's and 1890's.
} 
need a way of deriving indefinite probabilities them from them. That is, they need a theory of what is called "direct inference". Theories of objective indefinite probability propose that statistical inference gives us knowledge of indefinite probabilities, and then direct inference gives us knowledge of definite probabilities. Reichenbach (1949) pioneered the theory of direct inference. The basic idea is that if we want to know the definite probability PROB $(F a)$, we look for the logically strongest reference property $G$ such that we know the indefinite probability prob $(F x / G x)$ and we know $G a$, and then we identify $\operatorname{PROB}(F a)$ with $\operatorname{prob}(F x / G x)$. The latter is a kind of "total evidence" requirement. When we make direct inferences, we should appeal to probabilities that take account of the most information for which we know the relevant probabilities. For example, actuarial reasoning aimed at setting insurance rates proceeds in roughly this fashion. Kyburg (1974) was the first to attempt to provide firm logical foundations for direct inference. Some more recent attempts are those of Bacchus (1990), Halpern (1990), Pollock (1990), and Bacchus et al (1996).

Our purpose is to analyze the Sleeping Beauty problem from the perspective of objective probabilities and direct inference. For this, the details of the particular objective probability theory employed make little difference.

\section{The Objective Argument}

With our account of objective probabilities in place, turn to Sleeping Beauty.

On Sunday at brunch, Sleeping Beauty learns that she will undergo the following experiment. On Sunday night some experiments will put Sleeping Beauty to sleep. On Monday morning, the experimenters toss a fair coin, and then they awaken Beauty. Some time later that day they tell Beauty that it is Monday, and put her back to sleep. If the coin landed heads, the experimenters do nothing. If the coin landed tails, they administer a drug that erases her memories from Monday. On Tuesday, Beauty is either drugged up or sober. If drugged, she won't remember her Monday awakening; if sober, she will.

Beauty wakes up and doesn't remember a previous awakening. Then she considers this question: what is the probability that the coin landed heads? How should Beauty answer?

Let a "Sleeping Beauty scenario" be a particular instance of the Sleeping Beauty Problem. B $(t, s)$ means " $s$ is a Sleeping Beauty scenario, and $t$ is a time during $s$ ", and Toss $(x, s)$ means " $x$ is a (the) coin toss involved in $s^{\prime \prime}$. We can suppose that the times involved in a Sleeping Beauty scenario begin with Sunday (before Sleeping Beauty is put to sleep) and extend for 72 hours - through the following Tuesday. (The length of this period will turn out to be irrelevant.) Where $x$ is a coin toss, $\mathrm{H} x$ means " $x$ lands heads". The description of the chance set-up dictates that the coin is fair:

$$
\operatorname{prob}(\mathrm{H} x / \mathrm{B}(t, s) \& \operatorname{Toss}(x, s))=1 / 2 .
$$

Note that $x, s$, and $t$ are free variables in the formulas following "prob". Let $\sigma$ be a particular Sleeping Beauty scenario, and let $\tau$ be the toss of the coin in $\sigma$. On Sunday, Sleeping Beauty knows $\mathrm{B}($ now,$\sigma)$ \& Toss $(\tau, \sigma)$, but she knows nothing else that will give her a different probability. So at that time Sleeping Beauty can infer by direct inference that $\operatorname{PROB}(\mathrm{H} \tau)=1 / 2$.

Sleeping Beauty is put to sleep, the coin is tossed, and then at some subsequent time she is awakened and does not remember any previous awakening during the scenario. There is a time interval $\Delta$ such that, were she to reflect on the fact that she just awoke, her best estimate of when she awoke would be that it was within $\Delta$. For instance, she might think of $\Delta$ as "between 10 and 11 minutes ago". Let $W(t, s)$ mean "Sleeping Beauty awoke in the scenario $s$ sometime during the interval $\Delta$ (relative to $t$ ) and did not remember any previous awakening during $s$ ". When Sleeping Beauty awakes, what should she take the value of $\operatorname{PROB}(\mathrm{H} \tau)$ to be? She has learned something new, namely $\mathrm{W}($ now, $\sigma)$. If the probability

$\operatorname{prob}(\mathrm{H} x / \mathrm{W}(t, s) \& \mathrm{~B}(t, s) \& \operatorname{Toss}(x, s))$ 
is different from the probability in (1), she should make her direct inference from (2) rather than (1) because (2) involves a more specific reference property. So what is the value of the probability (2)?

The objective probability theorist can argue as follows. Let $\delta$ be the width of the interval $\Delta$ expressed in hours. Assuming a uniform probability distribution over times,

$$
\operatorname{prob}(\mathrm{W}(t, s) / \sim \mathrm{H} x \& \mathrm{~B}(t, s) \& \operatorname{Toss}(x, s))=(2 \times \delta) / 72
$$

because for each Sleeping Beauty scenario, if the coin toss lands tails then there are two intervals of width $\delta$ out of the 72 hour period of the scenario in which $\mathrm{W}(t, s)$ is true. Similarly,

$$
\operatorname{prob}(\mathrm{W}(t, s) / \mathrm{H} x \& \mathrm{~B}(t, s) \& \operatorname{Toss}(x, s))=\delta / 72 .
$$

It follows from (3) and (4) that:

$$
\operatorname{prob}(\mathrm{W}(t, s) / \sim \mathrm{H} x \& \mathrm{~B}(t, s) \& \operatorname{Toss}(x, s))=2 \times \operatorname{prob}(\mathrm{W}(t, s) / \mathrm{H} x \& \mathrm{~B}(t, s) \& \operatorname{Toss}(x, s)) .
$$

By two applications of Bayes' theorem:

$$
\begin{aligned}
& \operatorname{prob}(\sim \mathrm{H} x / \mathrm{W}(t, s) \& \mathrm{~B}(t, s) \& \operatorname{Toss}(x, s)) \\
& =\operatorname{prob}(\mathrm{W}(t, s) / \sim \mathrm{H} x \& \mathrm{~B}(t, s) \& \operatorname{Toss}(x, s)) \times \frac{\operatorname{prob}(\sim H x / B(t, s) \& \operatorname{Toss}(x, s))}{\operatorname{prob}(W(t, s) / B(t, s) \& \operatorname{Tos}(x, s))} \quad \text { (Bayes' theorem) } \\
& =2 \times \operatorname{prob}(\mathrm{W}(t, s) / \mathrm{H} x \& \mathrm{~B}(t, s) \& \operatorname{Toss}(x, s)) \times \frac{\operatorname{prob}(\sim H x / B(t, s) \& \operatorname{Toss}(x, s))}{\operatorname{prob}(W(t, s) / B(t, s) \& \operatorname{Tos}(x, s))} \quad \text { (from (5)) } \\
& =2 \times \operatorname{prob}(\mathrm{H} x / \mathrm{W}(t, s) \& \mathrm{~B}(t, s) \& \operatorname{Toss}(x, s)) \times \\
& \quad \frac{\operatorname{prob}(W(t, s) / B(t, s) \& \operatorname{Toss}(x, s))}{\operatorname{prob}(H x / B(t, s) \& \operatorname{Toss}(x, s))} \times \frac{\operatorname{prob}(\sim H x / B(t, s) \& \operatorname{Toss}(x, s))}{\operatorname{prob}(W(t, s) / B(t, s) \& \operatorname{Toss}(x, s))} \\
& =2 \times \operatorname{prob}(\mathrm{H} x / \mathrm{W}(t, s) \& \mathrm{~B}(t, s) \& \operatorname{Toss}(x, s)) \times \frac{\operatorname{prob}(\sim H x / B(t, s) \& \operatorname{Toss}(x, s))}{\operatorname{prob}(H x / B(t, s) \& \operatorname{Tos}(x, s))} \quad \text { (Bayes' theorem) }
\end{aligned}
$$

By (1), $\operatorname{prob}(\mathrm{H} x / \mathrm{B}(t, s) \& \operatorname{Toss}(x, s))=\operatorname{prob}(\sim \mathrm{H} x / \mathrm{B}(t, s) \& \operatorname{Toss}(x, s))$, so

$$
\operatorname{prob}(\sim \mathrm{H} x / \mathrm{W}(t, s) \& \mathrm{~B}(t, s) \& \operatorname{Toss}(x, s))=2 \times \operatorname{prob}(\mathrm{H} x / \mathrm{W}(t, s) \& \mathrm{~B}(t, s) \& \operatorname{Toss}(x, s))
$$

and hence

$$
\operatorname{prob}(\mathrm{H} x / \mathrm{W}(t, s) \& \mathrm{~B}(t, s) \& \operatorname{Toss}(x, s))=1 / 3 .
$$

Upon awakening, Sleeping Beauty learns $\mathrm{W}(n o w, \sigma) \& \mathrm{~B}(n o w, \sigma) \& \operatorname{Toss}(\tau, \sigma)$. Hence she can then infer by direct inference that:

$$
\operatorname{PROB}(\mathrm{H} \tau)=1 / 3 \text {. }
$$

Thus the objective probability theorist has a simple argument, by direct inference, for thirdism.

\section{Generalizing the Problem}

Most of the arguments in the literature arrive at the same conclusion as the objectivist argument, so the objectivist argument does not show that those arguments are wrong. But let us consider a generalization of the Sleeping Beauty problem. Suppose we use a biased coin to decide 
when Sleeping Beauty will be awakened. The objective argument handles this variant without difficulty. Suppose the probability of heads is $\alpha$. We get, as in (6) above:

$$
\begin{aligned}
& \operatorname{prob}(\sim \mathrm{H} x / \mathrm{W}(t, s) \& \mathrm{~B}(t, s) \& \operatorname{Toss}(x, s)) \\
& =2 \times \operatorname{prob}(\mathrm{H} x / \mathrm{W}(t, s) \& \mathrm{~B}(t, s) \& \operatorname{Toss}(x, s)) \times \frac{\operatorname{prob}(\sim H x / B(t, s) \& \operatorname{Tos} s(x, s))}{\operatorname{prob}(H x / B(t, s) \& \operatorname{Toss}(x, s))} \\
& =2 \times \operatorname{prob}(\mathrm{H} x / \mathrm{W}(t, s) \& \mathrm{~B}(t, s) \& \operatorname{Toss}(x, s)) \times \frac{1-\alpha}{\alpha}
\end{aligned}
$$

and hence

$$
\operatorname{prob}(\mathrm{H} x / \mathrm{W}(t, s) \& \mathrm{~B}(t, s) \& \operatorname{Toss}(x, s))=\frac{\alpha}{2-\alpha} .
$$

Then by direct inference

$$
\operatorname{PROB}(\mathrm{H} \tau)=\frac{\alpha}{2-\alpha} .
$$

So in this case we do not get the thirder answer. For instance, if $\alpha=2 / 3$, this has the consequence that $\operatorname{PROB}(\mathrm{H} \tau)=1 / 2$.

Can any of the other arguments in the literature handle this variant of the Sleeping Beauty problem with equal aplomb? It is unclear to us that they can, so let us issue this as a challenge. Regardless of whether other approaches can arrive at the same answer, it seems to us that the objectivist argument settles the matter as to what the correct answer is.

\section{Conclusion}

At long last, the party is over. It's time for Sleeping Beauty to rest.

Acknowledgement

This work was supported by NSF grant no. IIS-0412791.

\section{References}

Arntzenius, F.

2003 "Some problems for conditionalization and reflection." Journal of Philosophy 100: 356-370.

Bacchus, Fahiem

1990 Representing and Reasoning with Probabilistic Knowledge, MIT Press.

Bacchus, Fahiem, Adam J. Grove, Joseph Y. Halpern, Daphne Koller

1996 "From statistical knowledge bases to degrees of belief", Artificial Intelligence 87, 75-143.

de Finetti, Bruno

Bradley, D.

2003 "Sleeping Beauty: a note on Dorr's argument for 1/3." Analysis 63: 266-267.

Braithwaite, R. B.

1953 Scientific Explanation. Cambridge: Cambridge University Press.

Dorr, C.

2002 "Sleeping Beauty: in defense of Elga." Analysis 62: 53-62.

Elga, A.

2000 "Self-locating belief and the Sleeping Beauty problem." Analysis 60.2: 143-147. 
Fisher, R. A.

1922 "On the mathematical foundations of theoretical statistics." Philosophical Transactions of the Royal Society A, 222, 309-368.

Halpern, J. Y.

1990 "An analysis of first-order logics of probability", Artificial Intelligence 46, 311-350.

Hitchcock, C.

2004 "Beauty and the bets." Synthese 139: 405-420.

Horgan, T.

2004 "Sleeping Beauty awakened: new odds at the dawn of the new day." Analysis 64.1: 10-21.

2007 "Synchronic Bayesian updating and the generalized Sleeping Beauty problem." Analysis 67.1:

50-59.

Kneale, William

1949 Probability and Induction. Oxford: Oxford University Press.

Kyburg, Henry, Jr.

1961 Probability and the Logic of Rational Belief. Middletown, Conn.: Wesleyan University Press.

1974 The Logical Foundations of Statistical Inference, Dordrecht: Reidel.

1974a "Propensities and probabilities." British Journal for the Philosophy of Science 25, 321-353.

Lewis, D.

2001 "Sleeping Beauty: reply to Elga." Analysis 61.3: 171-176.

Monton, B.

2002 "Sleeping Beauty and the forgetful Bayesian." Analysis 62: 47-53.

Pollock, J.

1990 Nomic Probability and the Foundations of Induction. Oxford University Press.

Popper, Karl

1956 "The propensity interpretation of probability." British Journal for the Philosophy of Science 10, 25-42.

1957 "The propensity interpretation of the calculus of probability, and the quantum theory." In Observation and Interpretation, ed. S. Körner, 65-70. New York: Academic Press.

1959 The Logic of Scientific Discovery, New York: Basic Books.

Reichenbach, Hans

1949 A Theory of Probability, Berkeley: University of California Press. (Original German edition 1935.)

Russell, Bertrand

1948 Human Knowledge: Its Scope and Limits. New York: Simon and Schuster.

Sklar, Lawrence

1970 "Is propensity a dispositional concept?" Journal of Philosophy 67, 355-366.

1973 "Unfair to frequencies." Journal of Philosophy 70, 41-52.

van Fraassen, Bas

1981 The Scientific Image. Oxford: Oxford University Press.

Venn, John

1888 The Logic of Chance, 3rd ed. London.

Weintraub, R.

2004 "Sleeping Beauty: a simple solution." Analysis 64: 8-10.

White, R.

2006 "The generalized Sleeping Beauty problem: a challenge for thirders." Analysis 66.2: 114-119. 